\title{
BOUND STATE SOLUTION TO SCHRODINGER EQUATION WITH HulThen Plus Exponential Coulombic Potential with Centrifugal Potential Barrier using Parametric NIKIFOROVUVAROV METHOD
}

\author{
Ituen .B.Okon ${ }^{1}$,Oyebola Popoola ${ }^{2}$ and Eno .E. Ituen ${ }^{1}$ \\ ${ }^{1}$ Department of Physics, University of Uyo, Uyo, Nigeria. \\ ${ }^{2}$ Department of Physics, University of Ibadan, Ibadan, Nigeria. \\ ${ }^{1}$ Department of Physics, University of Uyo, Uyo, Nigeria.
}

\begin{abstract}
In this work, we obtained an approximate bound state solution to Schrodinger with Hulthen plus exponential Coulombic potential with centrifugal potential barrier using parametric Nikiforov-Uvarov method. We obtained both the eigen energy and the wave functions to non-relativistic wave equations. We implement Matlab algorithm to obtained the numerical bound state energies for various values of adjustable screening parameter at various quantum state.. The developed potential reduces to Hulthen potential and the numerical bound state energy conform to that of existing literature.
\end{abstract}

\section{KEYWORDS}

Schrodinger equation Hulthen plus exponential Coulombic potential, Nikiforov-Uvarov method.

\section{INTRODUCTION}

Schrodinger wave equation constitute non -relativistic wave equation. The solutions to the equation can either be exact constituting the s-waves or approximate through engaging appropriate approximation to the centrifugal term. Exact and approximate solutions to the equation find practical application in many branches of physics. In high energy physics, the solution is used to describe hadrons as bound state. In atomic Physics, the solution is applicable in the study of binding energy while in molecular physics, the solution is relevant in studying atomic correlation function [1-8]. Hulthen potential is a short range potential basically used in studying bound state energies of diatomic molecules [9]. Coulombic potential is an oscillating potential which is strongly repulsive at short inter-nuclear distance and has negligible effect at large internuclear distance. There is a need to extend the repulsive effect to a large inter-nuclear distance . In this work, we developed a potential model called exponential Coulombic potential with centrifugal potential barrier so as to extend the repulsive interaction to large inter-nuclear distance. The repulsive effect increases with smaller values of adjustable parameter $\alpha$ in the exponential term. The Hulthen potential is added to stabilize and bring into equilibrium the decaying effect of the exponential function. However, the developed potential model works perfectly for both small and large inter nuclear distance. 
Various analytical techniques have been adopted by different researchers to provide exact and approximate solutions to relativistic and non-relativistic wave equations. These are super symmetric quantum mechanics, exact quantization, point canonical transformation, asymptotic iteration method, Nikiforov-Uvarov method, Formular method, tri diagonolisation method, factorization method etc [10-20]. Correspondingly, these method are used to solve wave equations with some considerable potentials like : Mie-type, Kratzer, Poschl-teller, Hulthen, Morse, Eckart, Tiet-Hua, Tiet-wei and many more[21-28]. This paper is organized as follows: section 2 provides the basic review of parametric Nikiforov-Uvarov method. Section 3 gives the analytical solution of schrodinger equation with the developed potential. Section 4 gives the numerical bound state energy for Schrodinger wave equation . All graphs were plotted using origin software while the numerical computations were obtained using matlab programming. The developed potential model is given by

$$
V(r)=-\frac{V_{0} e^{-2 \alpha r}}{\left(1-e^{-2 \alpha r}\right)}+V_{1}\left[\frac{1}{2}\left(\frac{a}{r}\right)^{2}-\frac{a e^{-\alpha r}}{r}\right]
$$

Where $V_{0}$ is a potential depth, $V_{1}$ is the dissociation energy. $a$ is the positive constant which is strongly repulsive at short distance and $\alpha$ is the screening parameter that increases the repulsive effect. The graph of this potential against the inter-nuclear distance with various values of the screening parameter $\quad \alpha=0.1,0.2,0.3,0.4$ and 0.5 is shown below.

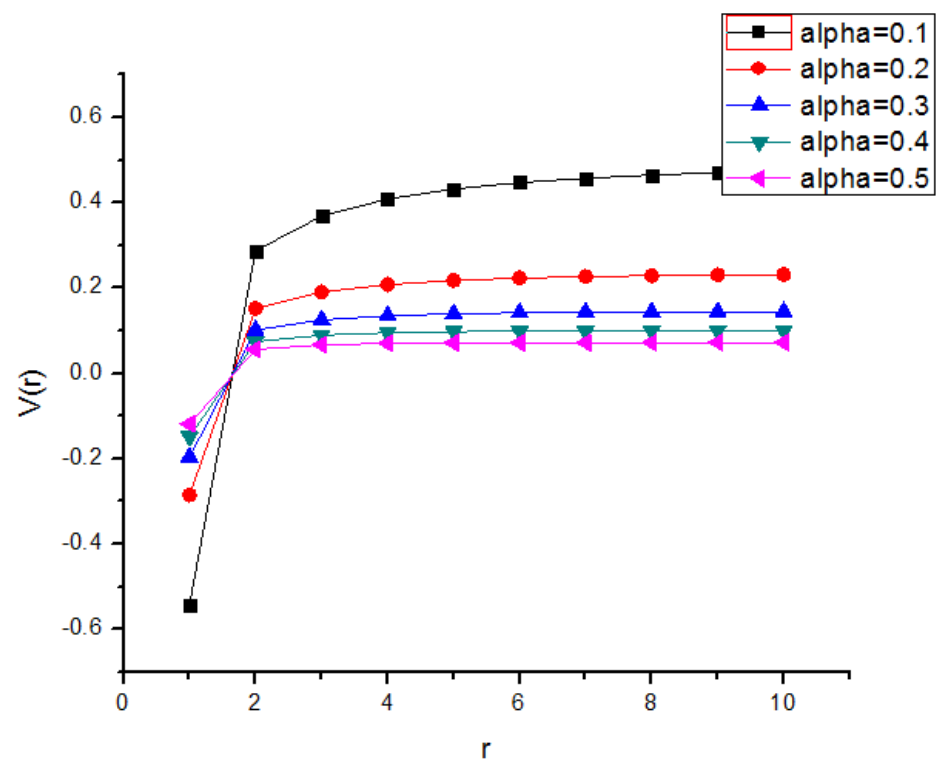

Figure 1

\section{Section 2: The Concept of Parametric Nikiforov-Uvarov Method}

Nikiforov-Uvarov method generally reduces second order partial differential equation to equation of hyper-geometric type and provide solutions in terms of orthogonal functions.[9] The parametric formalization of NU is applicable and valid for both central and non-central potential. Here, the hyper geometric differential equation is given by 
International Journal of Recent advances in Physics (IJRAP) Vol.5, No.2, May 2016

$$
\Psi^{\prime \prime}(s)+\frac{c_{1}-c_{2} s}{s\left(1-c_{3} s\right)} \Psi^{\prime}(s)+\frac{1}{s^{2}\left(1-c_{3} s\right)^{2}}\left[-\xi_{1} s^{2}+\xi_{2} s-\xi_{3}\right] \Psi(s)=o
$$

Where $c_{1}, c_{2}$ and $c_{3}$ are parametric constants. Other parametric constants are given below

$$
\left[\begin{array}{l}
c_{4}=\frac{1}{2}\left(1-c_{1}\right) \\
c_{5}=\frac{1}{2}\left(c_{2}-2 c_{3}\right) \\
c_{6}=c_{5}^{2}+\xi_{1} \\
c_{7}=2 c_{4} c_{5}-\xi_{2} \\
c_{8}=c_{4}^{2}+\xi_{3} \\
c_{9}=c_{3} c_{7}+c_{3}^{2} c_{8}+c_{6} \\
c_{10}=c_{1}+2 c_{4}+2 \sqrt{c_{8}} \\
c_{11}=c_{2}-2 c_{5}+2\left(\sqrt{c_{9}}+c_{3} \sqrt{c_{8}}\right) \\
c_{12}=c_{4}+\sqrt{c_{8}} \\
c_{13}=c_{5}-\left(\sqrt{c_{9}}+c_{3} \sqrt{c_{8}}\right)
\end{array}\right]
$$

The energy equation is given as

$c_{2} n-(2 n+1) c_{5}+(2 n+1)\left(\sqrt{c_{9}}+c_{3} \sqrt{c_{8}}\right)+n(n-1) c_{3}+c_{7}+2 c_{3} c_{8}+2 \sqrt{c_{8} c_{9}}=0$

The total wave function is the given by

$$
\Psi(s)=\phi(s) \chi_{n}(s)=N_{n} s^{c_{12}}\left(1-c_{3} s\right)^{c_{13}} P_{n}^{\left(c_{10}, c_{11}\right)}\left(1-2 c_{3} s\right)
$$

\section{SECTION 3: Radial SOlution OF Schrodinger Equation}

Schrodinger equation is given by

$$
\frac{d^{2} R}{d r^{2}}+\left[\frac{2 \mu}{\hbar^{2}}\left(E_{n l}-V(r)\right)-\frac{l(l+1)}{r^{2}}\right] R(r)=0
$$

Substituting equation (1) into (6) gives

$$
\frac{d^{2} R}{d r^{2}}+\left[\frac{2 \mu}{\hbar^{2}}\left(E_{n l}+\frac{V_{0} e^{-2 \alpha r}}{\left(1-e^{-2 \alpha r}\right)}-V_{1}\left(\frac{1}{2}\left(\frac{a}{r}\right)^{2}-\frac{a e^{-\alpha r}}{r}\right)\right)-\frac{l(l+1)}{r^{2}}\right] R(r)=0
$$

Let's define suitable approximation to the centrifugal term as 


$$
\frac{1}{r^{2}}=\frac{4 \alpha^{2} e^{-2 \alpha r}}{\left(1-e^{-2 \alpha r}\right)^{2}} \Rightarrow \frac{1}{r}=\frac{2 \alpha^{2} e^{-\alpha r}}{\left(1-e^{-2 \alpha r}\right)}
$$

Substituting equation (8) into (7) gives

$$
\frac{d^{2} R}{d r^{2}}+\left[\frac{2 \mu}{\hbar^{2}}\left(E_{n l}+\frac{V_{0} e^{-2 \alpha r}}{\left(1-e^{-2 \alpha r}\right)}-V_{1}\left(\frac{1}{2} \frac{4 a^{2} \alpha^{2} e^{-2 \alpha r}}{\left(1-e^{-2 \alpha r}\right)^{2}}-\frac{2 a \alpha e^{-2 \alpha r}}{\left(1-e^{-2 \alpha r}\right)}\right)\right)-\frac{4 \alpha^{2} l(l+1) e^{-2 \alpha r}}{\left(1-e^{-2 \alpha r}\right)^{2}}\right] R(r)=0
$$

In order to present equation (9) in a form that is conformable to equation (2) then, there is a need for the transformation

$$
s=e^{-2 \alpha r} \Rightarrow \frac{d^{2} R(r)}{d r^{2}}=4 \alpha^{2} e^{-4 \alpha r} \frac{d^{2} R(s)}{d s^{2}}+4 \alpha^{2} e^{-2 \alpha r} \frac{d R(s)}{d s}
$$

Substituting equation (10) into (9) and simplifying gives

$$
\begin{aligned}
& \frac{d^{2} R(s)}{d s^{2}}+\frac{(1-s)}{s(1-s)} \frac{d R(s)}{d s}+\frac{1}{s^{2}(1-s)^{2}}\left[\begin{array}{l}
-\left(\varepsilon^{2}+\delta^{2}+2 a \chi_{1} \alpha\right) s^{2} \\
+\left(2 \varepsilon^{2}+\delta^{2}-2 a^{2} \alpha^{2} \chi_{1}+2 a \alpha \chi_{1}-l(l+1)\right) s-\varepsilon^{2}
\end{array}\right] \\
& \text { Where } \varepsilon^{2}=\frac{-2 \mu E_{n l}}{4 \alpha^{2} \hbar^{2}}, \delta^{2}=\frac{2 \mu v_{0}}{4 \alpha^{2} \hbar^{2}}, \chi_{1}=\frac{2 \mu v_{1}}{4 \alpha^{2} \hbar^{2}}
\end{aligned}
$$

By comparing equation (11) to equation (2) then the following parametric constant is obtain

$$
\begin{aligned}
& \xi_{1}=\left(\varepsilon^{2}+\delta^{2}+2 a \chi_{1} \alpha\right) \\
& \xi_{2}=\left(2 \varepsilon^{2}+\delta^{2}-2 a^{2} \alpha^{2} \chi_{1}+2 a \alpha \chi_{1}-l(l+1)\right) \\
& \xi_{3}=\varepsilon^{2} \\
& c_{1}=c_{2}=c_{3}=1
\end{aligned}
$$


International Journal of Recent advances in Physics (IJRAP) Vol.5, No.2, May 2016

$$
\left[\begin{array}{l}
c_{4}=\frac{1}{2}\left(1-c_{1}\right)=0 \\
c_{5}=\frac{1}{2}\left(c_{2}-2 c_{3}\right)=-\frac{1}{2} \\
c_{6}=c_{5}^{2}+\xi_{1}=\frac{1}{4}+\varepsilon^{2}+\delta^{2}+2 a \chi_{1} \alpha \\
c_{7}=2 c_{4} c_{5}-\xi_{2}=-2 \varepsilon^{2}-\delta^{2}+2 a^{2} \alpha^{2} \chi_{1}-2 a \alpha \chi_{1}+l(l+1) \\
c_{8}=c_{4}^{2}+\xi_{3}=\varepsilon^{2} \\
c_{9}=c_{3} c_{7}+c_{3}^{2} c_{8}+c_{6}=\frac{1}{4}+2 a^{2} \alpha^{2} \chi_{1}+l(l+1) \\
c_{10}=c_{1}+2 c_{4}+2 \sqrt{c_{8}}=1+2 \varepsilon \\
c_{11}=c_{2}-2 c_{5}+2\left(\sqrt{c_{9}}+c_{3} \sqrt{c_{8}}\right)=2+2\left[\sqrt{\frac{1}{4}+2 a^{2} \alpha^{2} \chi_{1}+l(l+1)+\varepsilon}\right. \\
c_{12}=c_{4}+\sqrt{c_{8}}=\varepsilon \\
c_{13}=c_{5}-\left(\sqrt{c_{9}}+c_{3} \sqrt{c_{8}}\right)=-\frac{1}{2}-\left[\sqrt{\frac{1}{4}+2 a^{2} \alpha^{2} \chi_{1}+l(l+1)}+\varepsilon\right]
\end{array}\right.
$$

\subsection{Calculation of Energy}

The energy can be calculated by substituting equation (14) and (13) into (4) with simple mathematical algebraic simplification. Hence, the energy eigen equation become

$$
\left\{\begin{array}{l}
\left(n^{2}+n+\frac{1}{2}\right)+l(l+1)-\delta^{2}+2 a^{2} \alpha^{2} \chi_{1}-2 a \alpha \chi_{1}+(2 n+1)\left[\sqrt{\frac{1}{4}+2 a^{2} \alpha^{2} \chi_{1}+l(l+1)}+\varepsilon\right] \\
+2\left[\sqrt{\varepsilon^{2}\left(\frac{1}{4}+2 a^{2} \alpha^{2} \chi_{1}+l(l+1)\right)}\right]
\end{array}\right\}=0
$$

Factorising and rearranging equation (15) gives

$$
\varepsilon=-\left\{\frac{\left[\left(n^{2}+n+\frac{1}{2}\right)+l(l+1)-\delta^{2}+2 a^{2} \alpha^{2} \chi_{1}-2 a \alpha \chi_{1}\right]+(2 n+1)\left[\sqrt{\frac{1}{4}+2 a^{2} \alpha^{2} \chi_{1}+l(l+1)}+\varepsilon\right]}{\left[(2 n+1)+2 \sqrt{\left(\frac{1}{4}+2 a^{2} \alpha^{2} \chi_{1}+l(l+1)\right.}\right]}\right\}=0(16)
$$


Substituting the parameters of equation (12) into equation (16) and simplifying gives the final energy equation of the proposed potential as

$$
E_{n l}=-\frac{2 \alpha^{2} \hbar^{2}}{\mu}\left\{\frac{\left[\left(n^{2}+n+\frac{1}{2}\right)+l(l+1)-\frac{\mu v_{0}}{2 \alpha^{2} \hbar^{2}}+\frac{a^{2} \mu v_{1}}{\hbar^{2}}-\frac{a \mu v_{1}}{\alpha \hbar^{2}}\right]+(2 n+1)\left[\sqrt{\frac{1}{4}+\frac{a^{2} \mu v_{1}}{\hbar^{2}}+l(l+1)}\right]}{\left.\left[(2 n+1)+2 \sqrt{\left(\frac{1}{4}+\frac{a^{2} \mu v_{1}}{\hbar^{2}}+l(l+1)\right.}\right)\right]}\right\}^{2}=0(17)
$$

\subsection{Deduction from Equation (17)}

If we set $v_{1}=0$ in equation (1), then the potential function reduces to Hulthen potential. Making the same substitution into equation (17), then the energy of Hulthen potential become

$$
E_{n l}=-\frac{2 \alpha^{2} \hbar^{2}}{\mu}\left\{\frac{\left[\left(n^{2}+n+\frac{1}{2}\right)+l(l+1)-\frac{\mu v_{0}}{2 \alpha^{2} \hbar^{2}}\right]+(2 n+1)\left[\sqrt{\frac{1}{4}+l(l+1)}\right]}{\left[(2 n+1)+2 \sqrt{\left(\frac{1}{4}+l(l+1)\right.}\right]}\right\}^{2}=0(18)
$$

Equation (18) can further be simplified to give the final energy for Hulthen potential as

$$
E_{n l}=-\frac{2 \alpha^{2} \hbar^{2}}{\mu}\left\{\frac{[(n+l+2)(n+l)+1]-\frac{\mu v_{0}}{2 \hbar^{2} \alpha^{2}}}{[2(n+l+1)]}\right\}^{2}=0
$$

However, for the sake of numerical computation, equation (19) will be presented in the form as reported in some of the existing literature so as to enhance numerical comparison.

Assuming that $\alpha=\frac{\beta}{2}$ and $\mu=m$.then equation (19) become

$$
E_{n l}=-\frac{\hbar^{2} \beta^{2}}{2 m}\left\{\frac{[(n+l+2)(n+l)+1]-\frac{2 m v_{0}}{\hbar^{2} \beta^{2}}}{[2(n+l+1)]}\right\}^{2}=0
$$


International Journal of Recent advances in Physics (IJRAP) Vol.5, No.2, May 2016

\subsection{The Wave Function for the Proposed Potential}

The wave function for the proposed potential can be calculated using equation (5) and expressed in term of Jacobi polynomial. Thus,

$$
\left.\left.\Psi_{n}(s)=N_{n}(s) S^{\varepsilon}(1-s)^{-\frac{1}{2}\left[\sqrt{\frac{1}{4}+2 a^{2} \alpha^{2} \chi_{1}+l(l+1)}+\mathcal{E}\right.}\right] P_{n}^{\left[(1+2 \varepsilon),\left(2+\left(2 \sqrt{\frac{1}{4}+2 a^{2} \alpha^{2} \chi_{1}+l(l+1)}+\varepsilon\right)\right)\right.}\right](1-2 s) .
$$

$N_{n}$ is normalization constant and all other parameters have their usual meanings .

\section{SECTION 4: NUMERICAL COMPUTATION FOR ENERgY EQUATION OF THE Proposed Potential Using Schrodinger EQUation}

All programming were carried out using MATLAB software while graphical plot were carried out using origin software.

The energy equation for the proposed potential is given in equation (17). The computing parameters are:

$$
\begin{aligned}
& v_{0}=v_{1}=0.1, a=0.01, \\
& \alpha=(0.1,0.2,0.3,0.4 \text { and } 0.5) \\
& \hbar=m=\mu=1
\end{aligned}
$$

\begin{tabular}{|c|c|c|c|c|c|c|c|c|c|c|}
\hline \multicolumn{11}{|c|}{ NUMERICAL COMPUTATION FOR $\alpha=0.1$} \\
\hline$n$ & 1 & $E_{n}(e I)$ & $n$ & $\prod$ & $E_{n}(e V)$ & $n$ & $E_{n}(\mathrm{eV})$ & $n$ & ? & $E_{n}(\mathrm{eV})$ \\
\hline 0 & 0 & -0.803980900522 & 0 & l & -0.0127508708328 & 0 & \begin{tabular}{l|l}
2 & -0.0884454140736
\end{tabular} & 0 & 3 & .0 .377438327904 \\
\hline l & 0 & -0.012750112527 & l & 1 & -0.0884456901226 & 1 & \begin{tabular}{l|l}
2 & -0.377438534060
\end{tabular} & 1 & 3 & .0 .799200885610 \\
\hline 2 & 0 & -0.0 .88447070355 & 2 & 1 & -0.377439015098 & 2 & \begin{tabular}{|l|l}
2 & -0.799201159840
\end{tabular} & 2 & 3 & -0.133386209055 \\
\hline 3 & 0 & .0 .37744142028 & 3 & l & -0.799201799728 & 3 & -0.13338624267 & 3 & 3 & .0 .197461333650 \\
\hline 4 & 0 & -0.799204999158 & 4 & l & -0.133386321125 & 4 & -0.19746137323 & 4 & 3 & -0.271861058900 \\
\hline 5 & 0 & -0.13338671337 & 5 & 1 & -0.197461465586 & 5 & -0.27186110433 & 5 & 3 & -0.356449516970 \\
\hline 6 & 0 & -0.19746192736 & 6 & $\mathrm{l}$ & -0.271861210342 & 6 & -0.35644956820 & 6 & 3 & -0.451155147500 \\
\hline 7 & 0 & -0.27186174040 & 7 & $\mathrm{l}$ & -0.356449687740 & 7 & -0.45115520449 & 7 & 3 & .0 .555937351094 \\
\hline 8 & 0 & -0.35645028543 & 8 & $\mathrm{l}$ & -0.451155337494 & 8 & -0.55593741383 & 8 & 3 & .0 .670771702476 \\
\hline
\end{tabular}


International Journal of Recent advances in Physics (IJRAP) Vol.5, No.2, May 2016

NUMERICAL COMPUTATION FOR $\alpha=0.2$

\begin{tabular}{|r|r|c|r|l|l|l|l|r|r|r|r|}
\hline$n$ & $l$ & $E_{n}(\mathrm{eV})$ & $n$ & $l$ & $E_{n}(\mathrm{eV})$ & $n$ & $l$ & $E_{n}(\mathrm{eV})$ & $n$ & $l$ & $E_{n}(\mathrm{eV})$ \\
\hline $\mathbf{0}$ & $\mathbf{0}$ & -0.130027000374 & $\mathbf{0}$ & $\mathbf{l}$ & -0.37675365416 & $\mathbf{0}$ & $\mathbf{2}$ & -0.1333002908 & $\mathbf{0}$ & $\mathbf{3}$ & -0.271769008418 \\
\hline $\mathbf{l}$ & $\mathbf{0}$ & -0.376758462441 & $\mathbf{l}$ & $\mathbf{l}$ & -0.133300447778 & $\mathbf{1}$ & $\mathbf{2}$ & -0.27176909928 & $\mathbf{1}$ & $\mathbf{3}$ & -0.451060304997 \\
\hline $\mathbf{2}$ & $\mathbf{0}$ & -0.13330123221 & $\mathbf{2}$ & $\mathbf{l}$ & -0.271769311301 & $\mathbf{2}$ & $\mathbf{2}$ & -0.45106041899 & $\mathbf{2}$ & $\mathbf{3}$ & -0.670675356338 \\
\hline $\mathbf{3}$ & $\mathbf{0}$ & -0.27177037139 & $\mathbf{3}$ & $\mathbf{l}$ & -0.45106068498 & $\mathbf{3}$ & $\mathbf{2}$ & -0.67067549330 & $\mathbf{3}$ & $\mathbf{3}$ & -0.93044326708 \\
\hline $\mathbf{4}$ & $\mathbf{0}$ & -0.45106201494 & $\mathbf{4}$ & $\mathbf{l}$ & -0.67067581291 & $\mathbf{4}$ & $\mathbf{2}$ & -0.93044342697 & $\mathbf{4}$ & $\mathbf{3}$ & -1.23029265229 \\
\hline $\mathbf{5}$ & $\mathbf{0}$ & -0.67067741095 & $\mathbf{5}$ & $\mathbf{l}$ & -0.93044380006 & $\mathbf{5}$ & $\mathbf{2}$ & -1.23029283506 & $\mathbf{5}$ & $\mathbf{3}$ & -1.57018940923 \\
\hline $\mathbf{6}$ & $\mathbf{0}$ & -.930445665488 & $\mathbf{6}$ & $\mathbf{l}$ & -1.23029326156 & $\mathbf{6}$ & $\mathbf{2}$ & -1.57018961489 & $\mathbf{6}$ & $\mathbf{3}$ & -1.95011557634 \\
\hline $\mathbf{7}$ & $\mathbf{0}$ & -1.2302953940 & $\mathbf{7}$ & $\mathbf{1}$ & -1.57019009476 & $\mathbf{7}$ & $\mathbf{2}$ & -1.95011580487 & $\mathbf{7}$ & $\mathbf{3}$ & -2.37006096324 \\
\hline $\mathbf{8}$ & $\mathbf{0}$ & -1.57019249417 & $\mathbf{8}$ & $\mathbf{l}$ & -1.95011633811 & $\mathbf{8}$ & $\mathbf{2}$ & -2.37006121462 & $\mathbf{8}$ & $\mathbf{3}$ & -2.83001943916 \\
\hline
\end{tabular}

Table 2

NUMERICAL COMPUTATION FOR $\alpha=0.3$

\begin{tabular}{|c|c|c|c|c|c|c|c|c|c|c|c|}
\hline$n$ & $l$ & $E_{n}(\mathrm{eV})$ & $n$ & $l$ & $E_{n}(\mathrm{eV})$ & \multicolumn{1}{|c|}{$n$} & $l$ & $E_{n}(\mathrm{eV})$ & $n$ & $l$ & $E_{n}(\mathrm{eV})$ \\
\hline $\mathbf{0}$ & $\mathbf{0}$ & -0.087566744369 & $\mathbf{0}$ & $\mathbf{l}$ & -0.01332146021 & $\mathbf{0}$ & $\mathbf{2}$ & -0.35626232186 & $\mathbf{0}$ & $\mathbf{3}$ & -0.670579017134 \\
\hline $\mathbf{l}$ & $\mathbf{0}$ & -0.13321577873 & $\mathbf{l}$ & $\mathbf{l}$ & -0.356262680477 & $\mathbf{l}$ & $\mathbf{2}$ & -0.67057922259 & $\mathbf{l}$ & $\mathbf{3}$ & -1.07526288476 \\
\hline $\mathbf{2}$ & $\mathbf{0}$ & -0.35626447351 & $\mathbf{2}$ & $\mathbf{l}$ & -0.670579702013 & $\mathbf{2}$ & $\mathbf{2}$ & -1.07526314178 & $\mathbf{2}$ & $\mathbf{3}$ & -1.57009121724 \\
\hline $\mathbf{3}$ & $\mathbf{0}$ & -0.67058209904 & $\mathbf{3}$ & $\mathbf{l}$ & -1.07526374146 & $\mathbf{3}$ & $\mathbf{2}$ & -1.57009152573 & $\mathbf{3}$ & $\mathbf{3}$ & -2.15498775818 \\
\hline $\mathbf{4}$ & $\mathbf{0}$ & -1.07526673994 & $\mathbf{4}$ & $\mathbf{l}$ & -1.57009224555 & $\mathbf{4}$ & $\mathbf{2}$ & -2.15498811811 & $\mathbf{4}$ & $\mathbf{3}$ & -2.82992065438 \\
\hline $\mathbf{5}$ & $\mathbf{0}$ & -1.57009584464 & $\mathbf{5}$ & $\mathbf{l}$ & -2.15498895799 & $\mathbf{5}$ & $\mathbf{2}$ & -2.82992106577 & $\mathbf{5}$ & $\mathbf{3}$ & -3.59487468866 \\
\hline $\mathbf{6}$ & $\mathbf{0}$ & -2.15499315740 & $\mathbf{6}$ & $\mathbf{l}$ & -2.82992202567 & $\mathbf{6}$ & $\mathbf{2}$ & -3.59487515147 & $\mathbf{6}$ & $\mathbf{3}$ & -4.44984184624 \\
\hline $\mathbf{7}$ & $\mathbf{0}$ & -2.82992682526 & $\mathbf{7}$ & $\mathbf{l}$ & -3.59487623140 & $\mathbf{7}$ & $\mathbf{2}$ & -4.44984236050 & $\mathbf{7}$ & $\mathbf{3}$ & -5.39481758006 \\
\hline $\mathbf{8}$ & $\mathbf{0}$ & -3.59488163108 & $\mathbf{8}$ & $\mathbf{l}$ & -4.44984356043 & $\mathbf{8}$ & $\mathbf{2}$ & -5.39481814569 & $\mathbf{8}$ & $\mathbf{3}$ & -6.42979915438 \\
\hline
\end{tabular}

Table 3

NUMERICAL COMPUTATION FOR $\alpha=0.4$

\begin{tabular}{|c|c|c|c|c|c|c|c|c|c|c|c|}
\hline$n$ & l & $E_{n}(\mathrm{eV})$ & $n$ & l & $E_{n}(\mathrm{eV})$ & $n$ & $l$ & $E_{n}(\mathrm{eV})$ & $n$ & l & $E_{n}(\mathrm{eV})$ \\
\hline 0 & 0 & -0.37539441236 & 0 & l & -0.27158556005 & 0 & 2 & -0.67048295882 & 0 & 3 & -1.23009703893 \\
\hline 1 & 0 & -0.27158768013 & 1 & 1 & -0.67048359804 & 1 & 2 & -1.23009740451 & 1 & 3 & -1.94991866268 \\
\hline 2 & 0 & -0.67048679408 & 2 & l & -1.23009825749 & 2 & 2 & -1.94991911975 & 2 & 3 & -2.82982187135 \\
\hline 3 & 0 & -1.23010252247 & 3 & l & -1.94992018622 & 3 & 2 & -2.82982241985 & 3 & 3 & -3.86976359997 \\
\hline 4 & 0 & -1.94992551866 & 4 & l & -2.82982369974 & 4 & 2 & -3.86976423990 & 4 & 3 & -5.06972585981 \\
\hline 5 & 0 & -2.82983009918 & 5 & 1 & -3.86976573315 & 5 & 2 & -5.06972659117 & 5 & 3 & -6.42970005712 \\
\hline 6 & 0 & -3.86977319942 & 6 & l & -5.06972829779 & 6 & 2 & -6.42970087997 & 6 & 3 & -7.94968166570 \\
\hline 7 & 0 & -5.06973683082 & 7 & l & -6.42970279987 & 7 & 2 & -7.94968257997 & 7 & 3 & -9.62966811770 \\
\hline 8 & 0 & -6.42971239965 & 8 & 1 & -7.94968471322 & 8 & 2 & -9.62966912326 & 8 & 3 & -11.4696578679 \\
\hline
\end{tabular}

Table 4

NUMERICAL COMPUTATION FOR $\alpha=0.5$

\begin{tabular}{|c|c|c|c|c|c|c|c|c|c|c|c|}
\hline$n$ & l & $E_{n}(\mathrm{eV})$ & $n$ & $l$ & $E_{n}(\mathrm{eV})$ & $n$ & $l$ & $E_{n}(\mathrm{eV})$ & $n$ & l & $E_{n}(\mathrm{eV})$ \\
\hline 0 & 0 & -0.7960289798 & 0 & 1 & -0.45077678741 & 0 & 2 & -1.07506822147 & 0 & 3 & -1.94982020960 \\
\hline 1 & 0 & -0.45078011221 & 1 & 1 & -1.07506922097 & 1 & 2 & -1.94982078092 & 1 & 3 & -3.07470580563 \\
\hline 2 & 0 & -1.07507421838 & 2 & 1 & -1.94982211404 & 2 & 2 & -3.07470651984 & 2 & 3 & -4.44964382338 \\
\hline 3 & 0 & -1.94982877956 & 3 & 1 & -3.07470818636 & 3 & 2 & -4.44964468047 & 3 & 3 & -6.07460659180 \\
\hline 4 & 0 & -3.07471651908 & 4 & 1 & -4.44964668038 & 4 & 2 & -6.07460759175 & 4 & 3 & -7.94958255245 \\
\hline 5 & 0 & -4.44965667994 & 5 & 1 & -6.07460992500 & 5 & 2 & -7.94958369530 & 5 & 3 & -10.0745661834 \\
\hline 6 & 0 & -6.07462159135 & 6 & 1 & -7.94958636190 & 6 & 2 & -10.0745674691 & 6 & 3 & -12.4495545764 \\
\hline 7 & 0 & -7.94959969490 & 7 & 1 & -10.0745704690 & 7 & 2 & -12.4495560050 & 7 & 3 & -15.0745460816 \\
\hline 8 & 0 & -10.0745854688 & 8 & 1 & -12.4495593382 & 8 & 2 & -15.0745476529 & 8 & 3 & -17.9495397060 \\
\hline
\end{tabular}

Table 5 
International Journal of Recent advances in Physics (IJRAP) Vol.5, No.2, May 2016

Energy spectral diagram with Schrodinger equation for $\alpha=0.1$.

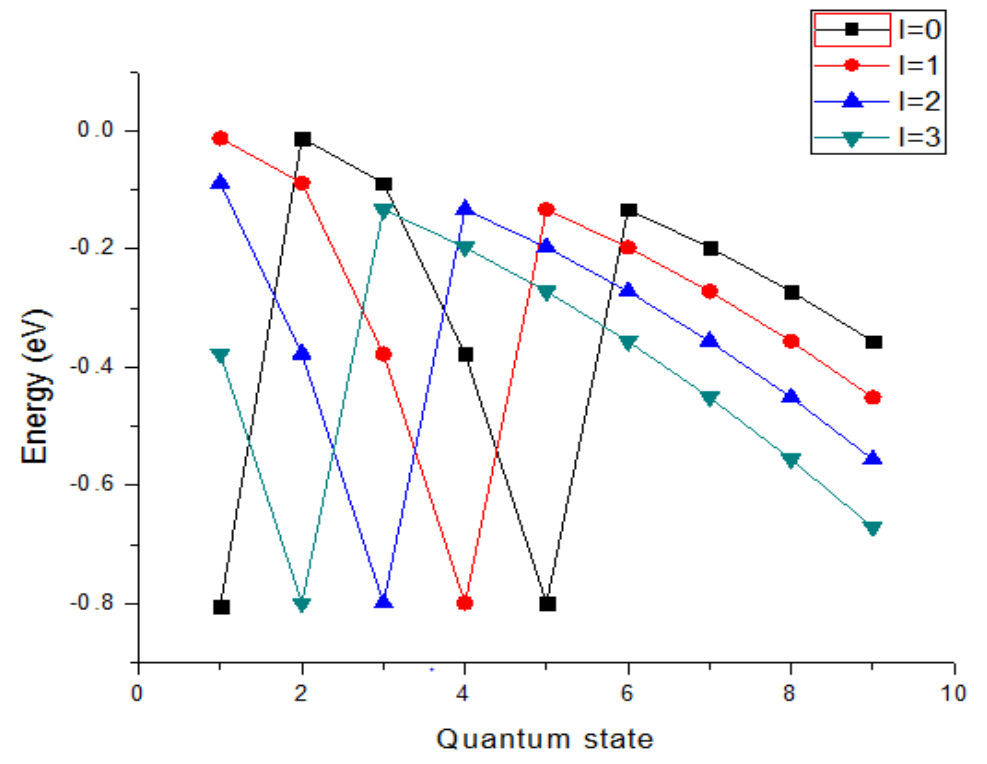

Figure 1

Energy spectral diagram with Schrodinger equation for $\alpha=0.2$.

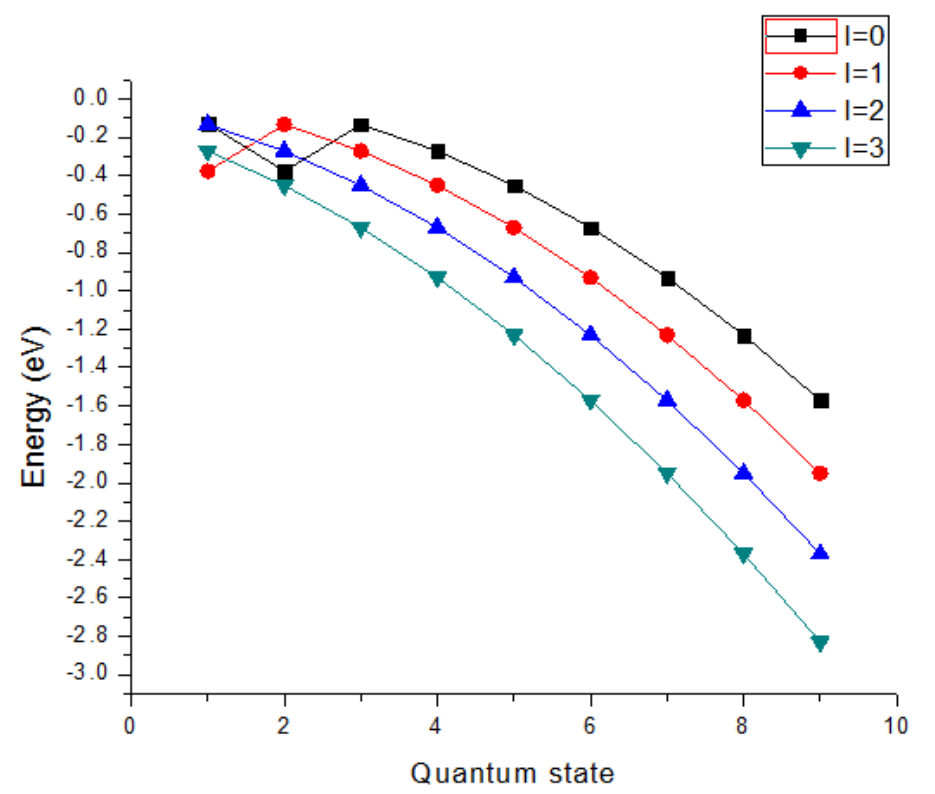

Figure 2 
International Journal of Recent advances in Physics (IJRAP) Vol.5, No.2, May 2016

Energy spectral diagram with Schrodinger equation for $\alpha=0.3$.

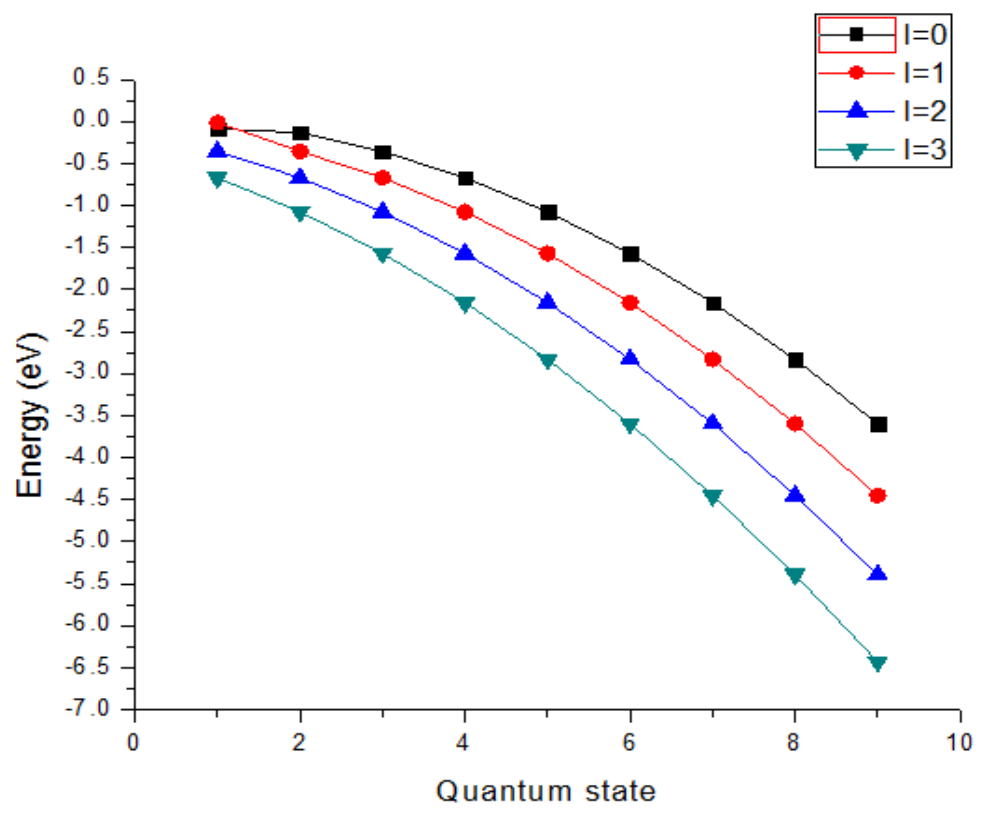

Figure 3

Energy spectral diagram with Schrodinger equation for $\alpha=0.4$.

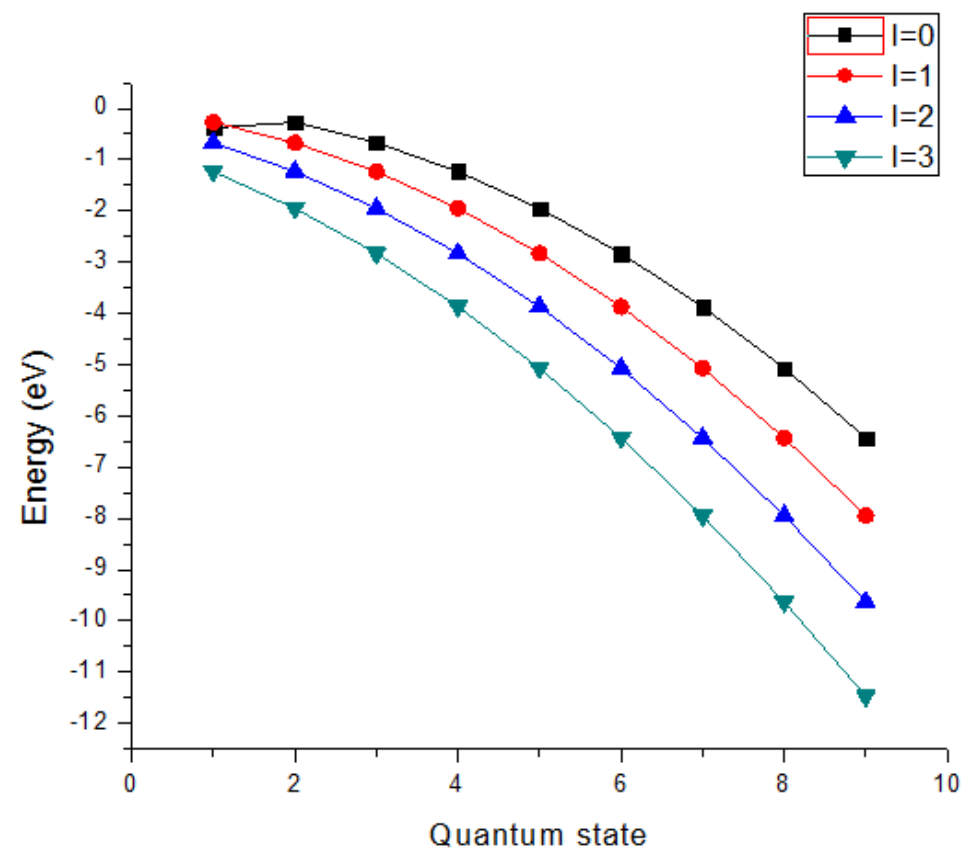

Figure 4 
Energy spectral diagram with Schrodinger equation for $\alpha=0.5$.

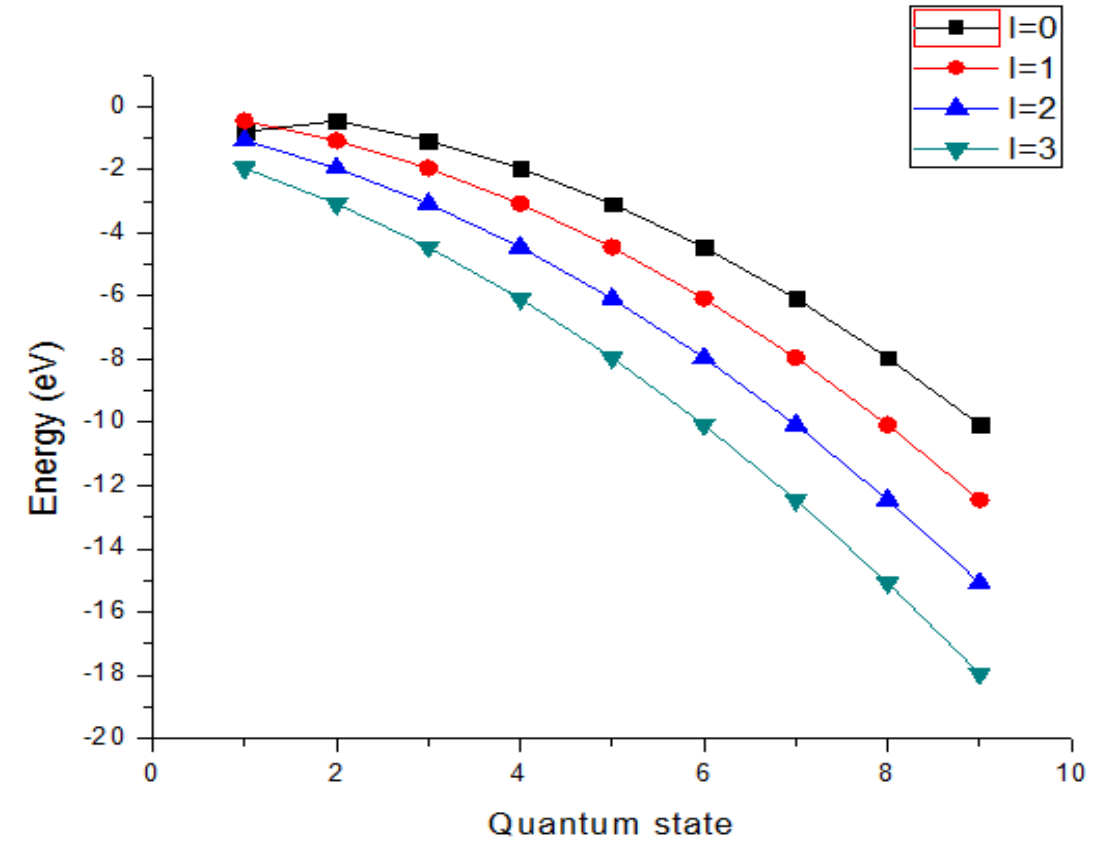

Figure 5

\section{SECTION 5: Discussion FOR Both TABLES AND FiguRES}

Numerical computation shows that the bound state energy with respect to Schrodinger equation decreases with an increase in quantum state. The energy also decreases with an increase in screening parameter $\alpha$.The energy spectral diagram shows a distinct quantum state indicating the quantisation of different energy levels as in figures (1-5). However, for $\alpha=0.1$ the energy spectral diagram shows a saw tooth wave form before quantisation indicating maximum oscillation of the charge particle with an initial velocity. We shall further investigate the energy spectral diagram of figure1 by engaging fourier transform analysis .

\subsection{Numerical Computation for Energy Equation of Hulthen Potential}

As stated earlier, the resulting potential reduces to hulthen potential. We adopted the parameter of (Arda and Serve, 2012) and (Fihho and Ricotta, 1995)for comparison. The existing literature computed for few quantum state, but we carried out our computations to accommodate higher quantum state. We carried out numerical computation for equation (20) using the following adopted parameters $\mu=m=1.0, v_{0}=\beta=\delta=(0.025,0.050,0.075,0.100$ and 0.150$)$. 
International Journal of Recent advances in Physics (IJRAP) Vol.5, No.2, May 2016

Bound state energy of Hulthen potential in comparison to other work.

\begin{tabular}{|l|l|l|l|l|ll|}
\hline$n$ & $l$ & $\delta$ & $\begin{array}{l}\text { Present } \\
\text { work }\end{array}$ & $\begin{array}{l}\text { (Arda } \\
\text { Server,2012) }\end{array}$ & $\begin{array}{l}\text { Filho } \\
\text { Ricotta, 1995) }\end{array}$ & and \\
\hline 0 & 1 & 0.025 & -0.1128125 & -0.1128130 & -0.1127600 \\
\hline 0 & 1 & 0.050 & -0.1012500 & -0.1012500 & -0.1010420 \\
\hline 0 & 1 & 0.075 & -0.0903125 & -0.0903120 & -0.0898450 \\
\hline 0 & 1 & 0.100 & -0.080000 & -0.0800000 & -0.0791700 \\
\hline 0 & 1 & 0.150 & -0.0612500 & -0.0612500 & -0.0594950 \\
\hline
\end{tabular}

Table 6

\subsection{Numerical Computation for Higher Quantum State of Hulthen Potential}

Bound state energy of Hulthen potential for $\delta=0.025$

\begin{tabular}{|c|c|c|c|c|c|c|c|c|c|c|c|}
\hline$n$ & $l$ & $E_{n}(\mathrm{eV})$ & $n$ & $l$ & $E_{n}(\mathrm{eV})$ & $n$ & $l$ & $E_{n}(\mathrm{eV})$ & $n$ & $l$ & $E_{n}(\mathrm{eV})$ \\
\hline $\mathbf{0}$ & $\mathbf{0}$ & -0.46320316000 & $\mathbf{0}$ & $\mathbf{l}$ & -0.112812510000 & $\mathbf{0}$ & $\mathbf{2}$ & -0.04882813000 & $\mathbf{0}$ & $\mathbf{3}$ & -0.0267382820000 \\
\hline $\mathbf{l}$ & $\mathbf{0}$ & -0.10408204000 & $\mathbf{l}$ & $\mathbf{l}$ & -0.043758683000 & $\mathbf{l}$ & $\mathbf{2}$ & -0.023247072000 & $\mathbf{l}$ & $\mathbf{3}$ & -0.0140281250000 \\
\hline $\mathbf{2}$ & $\mathbf{0}$ & -0.03896701700 & $\mathbf{2}$ & $\mathbf{l}$ & -0.020000001000 & $\mathbf{2}$ & $\mathbf{2}$ & -0.011628126000 & $\mathbf{2}$ & $\mathbf{3}$ & -0.0073003476000 \\
\hline $\mathbf{3}$ & $\mathbf{0}$ & -0.0169970710 & $\mathbf{3}$ & $\mathbf{l}$ & -0.009453125000 & $\mathbf{3}$ & $\mathbf{2}$ & -0.00564453150 & $\mathbf{3}$ & $\mathbf{3}$ & -0.0035220028000 \\
\hline $\mathbf{4}$ & $\mathbf{0}$ & -0.00750312600 & $\mathbf{4}$ & $\mathbf{l}$ & -0.004201389400 & $\mathbf{4}$ & $\mathbf{2}$ & -0.00242506400 & $\mathbf{4}$ & $\mathbf{3}$ & -0.0014111329000 \\
\hline $\mathbf{5}$ & $\mathbf{0}$ & -0.00297092040 & $\mathbf{5}$ & $\mathbf{l}$ & -0.001532206800 & $\mathbf{5}$ & $\mathbf{2}$ & -0.00076293950 & $\mathbf{5}$ & $\mathbf{3}$ & -0.0003481867300 \\
\hline $\mathbf{6}$ & $\mathbf{0}$ & -0.00084343120 & $\mathbf{6}$ & $\mathbf{l}$ & -0.000312500020 & $\mathbf{6}$ & $\mathbf{2}$ & -0.00007812500 & $\mathbf{6}$ & $\mathbf{3}$ & -0.0000031250001 \\
\hline $\mathbf{7}$ & $\mathbf{0}$ & -0.00005981445 & $\mathbf{7}$ & $\mathbf{1}$ & -0.000000964500 & $\mathbf{7}$ & $\mathbf{2}$ & -0.00006328125 & $\mathbf{7}$ & $\mathbf{3}$ & -0.0001865960800 \\
\hline $\mathbf{8}$ & $\mathbf{0}$ & -0.00011670525 & $\mathbf{8}$ & $\mathbf{0}$ & -0.000312500020 & $\mathbf{8}$ & $\mathbf{0}$ & -0.00054300105 & $\mathbf{8}$ & $\mathbf{0}$ & -0.0007834201700 \\
\hline
\end{tabular}

Table 7

Bound state energy of Hulthen potential for $\delta=0.050$

\begin{tabular}{|c|c|c|c|c|c|c|c|c|c|c|c|}
\hline$n$ & $l$ & $E_{n}(\mathrm{eV})$ & $n$ & $l$ & $E_{n}(\mathrm{eV})$ & \multicolumn{1}{|c|}{} & $l$ & $E_{n}(\mathrm{eV})$ & $n$ & $l$ & $E_{n}(\mathrm{eV})$ \\
\hline $\mathbf{0}$ & $\mathbf{0}$ & -0.427812520000 & $\mathbf{0}$ & $\mathbf{l}$ & -0.101250010000 & $\mathbf{0}$ & $\mathbf{2}$ & -0.042534728000 & $\mathbf{0}$ & $\mathbf{3}$ & -0.0225781260000 \\
\hline $\mathbf{l}$ & $\mathbf{0}$ & -0.08507813000 & $\mathbf{l}$ & $\mathbf{l}$ & -0.033368060000 & $\mathbf{l}$ & $\mathbf{2}$ & -0.01642578300 & $\mathbf{l}$ & $\mathbf{3}$ & -0.0091125010000 \\
\hline $\mathbf{2}$ & $\mathbf{0}$ & -0.025312502000 & $\mathbf{2}$ & $\mathbf{l}$ & -0.011250000000 & $\mathbf{2}$ & $\mathbf{2}$ & -0.005512500000 & $\mathbf{2}$ & $\mathbf{3}$ & -0.0028125000000 \\
\hline $\mathbf{3}$ & $\mathbf{0}$ & -0.007050782000 & $\mathbf{3}$ & $\mathbf{l}$ & -0.002812500000 & $\mathbf{3}$ & $\mathbf{2}$ & -0.001050347300 & $\mathbf{3}$ & $\mathbf{3}$ & -0.0003125000200 \\
\hline $\mathbf{4}$ & $\mathbf{0}$ & -0.001012500000 & $\mathbf{4}$ & $\mathbf{l}$ & -0.000138888900 & $\mathbf{4}$ & $\mathbf{2}$ & -0.000006377551 & $\mathbf{4}$ & $\mathbf{3}$ & -0.0001757812600 \\
\hline $\mathbf{5}$ & $\mathbf{0}$ & -0.00007812500 & $\mathbf{5}$ & $\mathbf{l}$ & -0.000516581700 & $\mathbf{5}$ & $\mathbf{2}$ & -0.0010986329000 & $\mathbf{5}$ & $\mathbf{3}$ & -0.0017013891000 \\
\hline $\mathbf{6}$ & $\mathbf{0}$ & -0.00184311240 & $\mathbf{6}$ & $\mathbf{l}$ & -0.002812500000 & $\mathbf{6}$ & $\mathbf{2}$ & -0.003707562000 & $\mathbf{6}$ & $\mathbf{3}$ & -0.0045125000000 \\
\hline $\mathbf{7}$ & $\mathbf{0}$ & -0.00531738300 & $\mathbf{7}$ & $\mathbf{l}$ & -0.006485339700 & $\mathbf{7}$ & $\mathbf{2}$ & -0.00750312600 & $\mathbf{7}$ & $\mathbf{3}$ & -0.0083910130000 \\
\hline $\mathbf{8}$ & $\mathbf{0}$ & -0.010034723000 & $\mathbf{8}$ & $\mathbf{0}$ & -0.011250000000 & $\mathbf{8}$ & $\mathbf{0}$ & -0.012295972000 & $\mathbf{8}$ & $\mathbf{0}$ & -0.0132031260000 \\
\hline
\end{tabular}


International Journal of Recent advances in Physics (IJRAP) Vol.5, No.2, May 2016

Bound state energy of Hulthen potential for $\delta=0.075$

\begin{tabular}{|c|c|c|c|c|c|c|c|c|c|c|c|}
\hline$n$ & $l$ & $E_{n}(\mathrm{eV})$ & $n$ & $l$ & $E_{n}(\mathrm{eV})$ & $n$ & $l$ & $E_{n}(\mathrm{eV})$ & $n$ & $l$ & $E_{n}(\mathrm{eV})$ \\
\hline $\mathbf{0}$ & $\mathbf{0}$ & -0.39382812000 & $\mathbf{0}$ & $\mathbf{l}$ & -0.09031250000 & $\mathbf{0}$ & $\mathbf{2}$ & -0.03667535000 & $\mathbf{0}$ & $\mathbf{3}$ & -0.01876953000 \\
\hline $\mathbf{l}$ & $\mathbf{0}$ & -0.06798828000 & $\mathbf{l}$ & $\mathbf{l}$ & -0.02438367900 & $\mathbf{l}$ & $\mathbf{2}$ & -0.01078613300 & $\mathbf{l}$ & $\mathbf{3}$ & -0.00525312450 \\
\hline $\mathbf{2}$ & $\mathbf{0}$ & -0.01459201300 & $\mathbf{2}$ & $\mathbf{l}$ & -0.00499999940 & $\mathbf{2}$ & $\mathbf{2}$ & -0.00165312480 & $\mathbf{2}$ & $\mathbf{3}$ & -0.00042534710 \\
\hline $\mathbf{3}$ & $\mathbf{0}$ & -0.00141113250 & $\mathbf{3}$ & $\mathbf{l}$ & -0.00007812495 & $\mathbf{3}$ & $\mathbf{2}$ & -0.00010633687 & $\mathbf{3}$ & $\mathbf{3}$ & -0.00057557410 \\
\hline $\mathbf{4}$ & $\mathbf{0}$ & -0.00052812515 & $\mathbf{4}$ & $\mathbf{l}$ & -0.00170138910 & $\mathbf{4}$ & $\mathbf{2}$ & -0.00294802340 & $\mathbf{4}$ & $\mathbf{3}$ & -0.00410644570 \\
\hline $\mathbf{5}$ & $\mathbf{0}$ & -0.00521050400 & $\mathbf{5}$ & $\mathbf{l}$ & -0.00715720750 & $\mathbf{5}$ & $\mathbf{2}$ & -0.00881958100 & $\mathbf{5}$ & $\mathbf{3}$ & -0.01023244800 \\
\hline $\mathbf{6}$ & $\mathbf{0}$ & -0.01320312600 & $\mathbf{6}$ & $\mathbf{l}$ & -0.01531250200 & $\mathbf{6}$ & $\mathbf{2}$ & -0.01706115300 & $\mathbf{6}$ & $\mathbf{3}$ & -0.01852812800 \\
\hline $\mathbf{7}$ & $\mathbf{0}$ & -0.02358521000 & $\mathbf{7}$ & $\mathbf{1}$ & -0.02562596800 & $\mathbf{7}$ & $\mathbf{2}$ & -0.02731953400 & $\mathbf{7}$ & $\mathbf{3}$ & -0.02874548500 \\
\hline $\mathbf{8}$ & $\mathbf{0}$ & -0.03592689700 & $\mathbf{8}$ & $\mathbf{0}$ & -0.03781250500 & $\mathbf{8}$ & $\mathbf{0}$ & -0.03939114500 & $\mathbf{8}$ & $\mathbf{0}$ & -0.04073134000 \\
\hline
\end{tabular}

Bound state energy of Hulthen potential for $\delta=0.100$

\begin{tabular}{|c|c|c|c|c|c|c|c|c|c|c|c|}
\hline$n$ & $l$ & $E_{n}(e V)$ & $n$ & $l$ & $E_{n}(\mathrm{eV})$ & $n$ & $l$ & $E_{n}(\mathrm{eV})$ & $n$ & $l$ & $E_{n}(\mathrm{eV})$ \\
\hline 0 & 0 & -0.361250000000 & 0 & 1 & -0.08000000600 & 0 & 2 & -0.031250004000 & 0 & 3 & -0.01531250100 \\
\hline 1 & 0 & -0.052812506000 & 1 & 1 & -0.01680555800 & 1 & 2 & -0.006328125500 & 1 & 3 & -0.00245000030 \\
\hline 2 & 0 & -0.006805556400 & 2 & 1 & -0.00125000010 & 2 & 2 & -0.000050000002 & 2 & 3 & -0.00013888890 \\
\hline 3 & 0 & -0.000078125006 & 3 & 1 & -0.00125000010 & 3 & 2 & -0.002812500000 & 3 & 3 & -0.00431122500 \\
\hline 4 & 0 & -0.006050000400 & 4 & 1 & -0.00888889000 & 4 & 2 & -0.011250000000 & 4 & 3 & -0.01320312600 \\
\hline 5 & 0 & -0.018368058000 & 5 & 1 & -0.02145408300 & 5 & 2 & -0.023925783000 & 5 & 3 & -0.02594135900 \\
\hline 6 & 0 & -0.034923470000 & 6 & 1 & -0.03781250000 & 6 & 2 & -0.040138893000 & 6 & 3 & -0.04205000400 \\
\hline 7 & 0 & -0.054863285000 & 7 & 1 & -0.05742284300 & 7 & 2 & -0.059512503000 & 7 & 3 & -0.06125000500 \\
\hline 8 & 0 & -0.077793220000 & 8 & 0 & -0.08000000600 & 8 & 0 & -0.081828510000 & 8 & 0 & -0.08336806000 \\
\hline
\end{tabular}

Table 10

Bound state energy of Hulthen potential for $\delta=0.150$

\begin{tabular}{|c|c|c|c|c|c|c|c|c|c|c|c|}
\hline$n$ & l & $E_{n}(\mathrm{eV})$ & $n$ & $l$ & $E_{n}(\mathrm{eV})$ & $n$ & $i$ & $E_{n}(\mathrm{eV})$ & $n$ & $l$ & $E_{n}(\mathrm{eV})$ \\
\hline 0 & 0 & -0.300312500000 & 0 & 1 & -0.061250000000 & 0 & 2 & -0.02170138800 & 0 & 3 & -0.009453125000 \\
\hline 1 & 0 & -0.028203122000 & 1 & 1 & -0.005868054500 & 1 & 2 & -0.00095703100 & 1 & 3 & -0.000012499977 \\
\hline 2 & 0 & -0.000034722158 & 2 & 1 & -0.001250000300 & 2 & 2 & -0.00361250040 & 2 & 3 & -0.005868056000 \\
\hline 3 & 0 & -0.010332032000 & 3 & 1 & -0.015312501000 & 3 & 2 & -0.01917534900 & 3 & 3 & -0.022200258000 \\
\hline 4 & 0 & -0.035112508000 & 4 & 1 & -0.040138897000 & 4 & 2 & -0.04393495600 & 4 & 3 & -0.046894540000 \\
\hline 5 & 0 & -0.068758690000 & 5 & 1 & -0.073016590000 & 5 & 2 & -0.07629395000 & 5 & 3 & -0.078892750000 \\
\hline 6 & 0 & -0.109445160000 & 6 & 1 & -0.112812510000 & 6 & 2 & -0.11546682600 & 6 & 3 & $6-0.117612496000$ \\
\hline 7 & 0 & -0.156450210000 & 7 & 1 & -0.158985330000 & 7 & 2 & -0.16102812000 & 7 & 3 & -0.162709190000 \\
\hline 8 & 0 & -0.209448310000 & 8 & 0 & -0.211249990000 & 8 & 0 & -0.21272984000 & 8 & 0 & -0.213967010000 \\
\hline
\end{tabular}


International Journal of Recent advances in Physics (IJRAP) Vol.5, No.2, May 2016

\section{Discussion of The Above Table}

Table 6 shows our numerical computation as compared to other work. Our result is in agreement to that of existing literature ascertaining the mathematical accuracy of the analytical solutions. Tables 7-11 show higher quantum state of table 6 for different values of $\delta$.

\section{CONCLUSION}

We engaged a powerful tool of Nikiforov-Uvarov method to compute bound state solution of non -relativistic wave equation. We developed a new potential model that showcase strong repulsive force both at short and large internuclear distance. This potential reduces to a well known potential called the Hulthen potential which is a short range oscillating potential. We also implement some algorithm to carried out numerical computation for the resulting energy equations for various values of screening parameter. Our analytic and computational result for Hulthen potential is in agreement to that of existing literature.

\section{ACKNOWLEDGEMENT}

The authors are grateful to the anonymous reviewer for his useful comment which have significantly help for the improvement of this article.

\section{REFERENCES}

[1] OkonI.B ,Ituen E.E, .PopoolaO.O and Antia A.D.(2013), “Analytical Solutions of Schrodinger Equation with Mie-Type Potential Using Factorisation Method", International Journal of Recent Advances In Physics, Vol.2, No.2, pp1-7.

[2] OkonI.B, Isonguyo C.N, Ituen, E.E and Ikot A.N. (2014), "Energy Spectrum for Some Diatomic Molecules with Generalized Manning-Rosen Potential Using Supersymmetric Quantum Mechanics (SUSY)".Conference proceedings of Nigerian Institute of Physics, 2014.

[3] Okon I. B, Popoola O.O and Isonguyo. C.N (2014), "Exact Bound state Solution of q-deformed Woods-Saxon plus modified Coulomb Potential Using Conventional Nikiforov-Uvarov Method", International Journal of Recent Advances in Physics Vol. 3, No.4.

[4] Okon I.B and Popoola. O.O.(2015), "Bound state solution of Schrodinger equation with Hulthen plus generalised exponential coulomb potential using Nikiforov-Uvarov method", International Journal of Recent Advances in Physics. Vol.4, No.3.Doi:10.14810/ijrap.2015.430

[5] Cheng .F.Y and Dai. T Q(2007), "Exact solution of the Schr" odinger equation for the modified Kratzer potential plus a ring-shaped potential by the Nikiforov-Uvarov method," PhysicaScripta, vol. 75 , no. 3, pp. 274-277

[6] Berkdemir. A. Han J, ,2006 "Bound state solutions of the Schr"odinger equation for modified Kratzer's molecular potential," Chemical Physics Letters, vol. 417, no. 4-6, pp. 326-329

[7] Isonguyo C. N, Okon, I. B and Ikot A. N (2013), "Semi-relativistic treatment of Hellmann potential using Super symmetric Quantum Mechanics", Journal of the Nigerian Association of Mathematical Physics (NAMP Journal). Vol.25, No. 2, pp121-126.

[8] Isonguyo, C. N ,. Okon I.B, Ikot A.N and Hassanabadi H. (2014) , "Solution of Klein-Gordon Equation for some diatomic molecules with New Generalised Morse-like potential Using SUSYQM", Bull. Korean Chem. Soc. 2014, Vol. 35, No. $12 \quad 3443$ http://dx.doi.org/10.5012/bkcs.2014.35.12.3443

[9] Nikiforov A.F, Uvarov V. B (1988), " Special Functions of Mathematical Physics,", Birkhauser, Bassel

[10] Dong S.H., Factorization Method in Quantum Mechanics. (2007). Springer-Verlag, Berlin

[11] Hassanabadi. H., Zarrinkamar, S. and Rahimov, H (2011) “ Approximate solution of D-dimensional Klein-Gordon equation with Hulthen-type potential via SUSYQM”. Commun.Theor. Phys. 56, 423 428 
International Journal of Recent advances in Physics (IJRAP) Vol.5, No.2, May 2016

[12] Boonserm, P. and Visser, M.(2011) Quasi-normal frequencies "Key analytic results. arXiv: 1005.4483v3 [math-ph]

[13] Ikhdair S. M. and Sever R.( 2007). "A perturbative treatment for the bound states of the Hellmann potential”, Journal of Molecular Structure, vol. 809, no. 1-3, pp. 103-113

[14] Berkdemir C., Berk demir A. and Sever R. ( 2006) "Systematical approach to the exact solution of the Dirac equation for a deformed form of the Woods-Saxon potential", Journal of Physics A, vol. 39, no. 43, pp. $13455-13463$.

[15] Fl“ugge S., Practical Quantum Mechanics, Springer- Verlag, Berlin (1994).

[16] R. Dutt R., Chowdhury. K and Varshni,Y.P (1985), "An improved calculation foscreened Coulomb potentials in Rayleigh-Schrodinger”, perturbation theory. J. Phys. A: Math. Gen. vol. 18, pp13791388 .

[17] Ikhdair S. M andSever R .(2007), "An alternative simple solution of the sextican harmonic oscillator and perturbed coulomb problems," International Journal of Modern Physics C, vol. 18, No10, pp1571.

[18] Oyewumi K.J, AkinpeluO.E and Agboola A.D (2008) "Exactly Complete Solutions of the Pseudoharmonic Potential in N-Dimensions," International Journal of Theoretical Physics, Vol. 47, No. 4, pp. 1039-1057(2008) doi:10.1007/s10773-007-9532-x.

[19] Hassanabadi H., Zarrinkamar S. and Rajabi A.A. (2011) "Exact solutions of D-dimensional schrödinger equation for an energy-dependent potential by NU method" Commun. Theor.Phys. Vol. 55 , pp 541.

[20] Ikot A.N, Antia A.D, Akpabio, L.E and Obu, A. J (2011), “Analytic Solutions of Schrodinger Equation with Two-Dimensional Harmonic Potential in Cartesian and Polar Coordinates via Nikiforov-Uvarov Method”, Journal of Vectorial Relativity, Vol. 6, No. 2, pp. 65-76.

[21] Greene R. L and Aldrich C. (1976). "Variational wave functions for a screened Coulomb potential", Physical Review A, vol. 14, no. 6, pp. 2363-2366. pp1-7.

[22] BeraP.K(2012)" The exact solutions for the interaction $V(r)=\alpha r^{2 d-2}-\beta r^{d-2}$ by NikiforovUvarov method" Pramana-J. Phys Vol. 78, pp667.

[23] Ikhdair S. M. and Sever R. ( 2011). "Relativistic and nonrelativistic bound states of the isotonic oscillator by Nikiforov-Uvarov method," Journal of Mathematical Physics, vol. 52, no. 12, article 122108.

[24] Fakhri H., Sadeghi J (2004) "Super symmetry approaches to the bound states of the generalized Woods-Saxon potential" Mod. Phys. Lett. A Vol. 19, pp615-625.

[25] Khounfais K., Boudjedaa T. and Chetouani L (2004), "Scattering matrix for Feshbach- Villars equation for spin 0 and 1/2: Woods-Saxon potential",Czech. J. Phys. Vol.54, pp697-792.

[26] Ikot A.N, Awoga O. A. Akpabio, L. E and Antia A. D (2011) “Exact Solutions of Schrodinger equation with Woods-Saxon Plus Rosen-Morse Potential”, Elixir vib. Spec.Vol. 2011, pp23032307.

[27] Arda. A and Sever R(2012). "Approximate Analytical solutions of a two Term diatomic molecular potential with centrifugal barrier. Arxiv: 1204. 126V[math-ph]

[28] E. D. Filho and R. M. Ricotta, Mod. Phys. Lett. A 10, 1613 (1995). 